\section{CardioRenal Medicine}

\title{
Apolipoprotein L1 Genetic Variants Are Associated with Chronic Kidney Disease but Not with Cardiovascular Disease in a Population Referred for Cardiac Catheterization
}

\author{
Hanghang Wang ${ }^{a}$ Patrick H. Pun ${ }^{b} \quad$ Lydia Kwee $^{a} \quad$ Damian Craig ${ }^{a}$ \\ Carol Haynes $^{a}$ Megan Chryst-Ladd ${ }^{a}$ Laura P. Svetkey ${ }^{b} \quad$ Uptal D. Patel ${ }^{b}$ \\ Elizabeth R. Hauser ${ }^{a}$ Martin R. Pollak ${ }^{d}$ William E. Kraus ${ }^{a, c}$ Svati H. Shah ${ }^{a, c}$ \\ ${ }^{a}$ Duke Molecular Physiology Institute, ${ }^{b}$ Division of Nephrology, Department of Medicine, \\ 'Division of Cardiology, Department of Medicine, Duke University School of Medicine, \\ Durham, NC, and ${ }^{\mathrm{d}}$ The Laboratory of Inherited Kidney Disease, Beth Israel Deaconess Medical \\ Center, Boston, MA, USA
}

\section{Keywords}

Apolipoprotein L1 · Chronic kidney disease - Cardiovascular disease - Gene polymorphism . Population genetics

\begin{abstract}
Background: While the association between APOL1 genetic variants and chronic kidney disease (CKD) has been established, their association with cardiovascular disease (CVD) is unclear. This study sought to understand CKD and cardiovascular risk conferred by APOL1 variants in a secondary cardiovascular prevention population. Methods: Two risk variants in APOL1 were genotyped in African-Americans $(n=1,641)$ enrolled in the CATHGEN biorepository, comprised of patients referred for cardiac catheterization at Duke University Hospital, Durham, NC, USA (2001-2010). Individuals were categorized as noncarriers $(n=722)$, heterozygote $(n=771)$, or homozygote carriers $(n=231)$ of APOL1 risk alleles. Multivariable logistic regression and Cox proportional hazards models adjusted for CVD risk factors were used to assess the association between $A P O L 1$ risk variants and prevalent and incident CKD, prevalent coronary artery disease (CAD), incident CVD events, and mortality. Results: The previously identified association between APOL1 variants and prevalent CKD was confirmed (OR: 1.85, 95\% CI: $1.33-2.57, p=0.0002$ ). No statistically significant associations were detected between APOL1 variants and incident CKD or prevalent CAD, incident CVD events or mortality. Age, type 2 diabetes, and ejection fraction at baseline were significant clinical factors that predicted the risk of incident CKD in a subgroup analysis of APOL1 homozygous individuals. Conclusion: APOL1 genetic variants are not associated with CAD or incident CVD events in a cohort of in-
\end{abstract}


Wang et al.: Apolipoprotein L1 Genetic Variants Are Associated with CKD but Not with CVD in a Population Referred for Cardiac Catheterization

dividuals with a high burden of cardiometabolic risk factors. In individuals with homozygous APOL1 status, factors that predicted subsequent CKD included age, presence of type 2 diabetes, and ejection fraction at baseline.

(C) 2016 S. Karger AG, Basel

\section{Background}

Racial disparities in the risk of developing cardiac and renal diseases have been attributed to environmental, socioeconomic, and genetic factors [1]. The presence of 2 genetic variants (G1 and G2) in the apolipoprotein L1 (APOL1) gene is strongly associated with increased susceptibility to developing chronic kidney disease (CKD) [2-6]. These variants are rare in individuals of European ancestry but common in African-Americans, with 50\% of AfricanAmericans harboring at least 1 risk allele and 10-15\% carrying 2 risk alleles [2]. The APOL1 gene encodes apolipoprotein L1, a protein involved in the lysis of the parasite Trypanosoma brucei that causes African sleeping sickness. The trypanolytic function may explain the increased allele frequency of G1 and G2 in individuals of African descent as a possible result of positive selection in recent evolutionary history [7].

Apart from the observed association between APOL1 risk variants and CKD, recent results from the Jackson Heart Study (JHS) and Women's Health Initiative (WHI) also reported an increased risk of cardiovascular disease (CVD) in African-Americans with APOL1 variants [6]. However, an increased CVD risk was not observed in other cohort studies of APOL1 carriers $[4,5,8]$, and no studies so far have examined this association in a secondary cardiovascular prevention population. Thus, to clarify and expand the understanding of APOL1 variants, we assessed the association of APOL1 variants with CKD, coronary artery disease (CAD), and incident CVD events in a secondary cardiovascular prevention population with a high incident CVD event rate, the CATHeterization GENetics (CATHGEN) study [9].

\section{Methods}

Study Cohort

The cardiac CATHGEN study is approved by the Duke University Institutional Review Board and consists of samples collected from 9,249 sequential consenting adult patients undergoing cardiac catheterization at Duke University Hospital, Durham, NC, USA, between 2001 and 2010 [9]. Consistent with previous studies $[2,5,10]$, we restricted our analysis to 1,641 African-Americans in CATHGEN.

Baseline and subsequent estimated glomerular filtration rates (eGFR) were calculated using the CKD-EPI equation [11], with creatinine measurements obtained on the day of catheterization and during the followup visits. Prevalent CKD was defined as baseline eGFR $<60 \mathrm{~mL} / \mathrm{min} / 1.73 \mathrm{~m}^{2}$; incident CKD was defined as no $\mathrm{CKD}$ at enrollment with at least 2 subsequent follow-up eGFR measurements $<60 \mathrm{~mL} / \mathrm{min} / 1.73 \mathrm{~m}^{2}$ and obtained at least 3 months apart. Prevalent CAD was defined as the presence of at least 1 diseased vessel ( $>50 \%$ occlusion) by angiography performed within 1 year of enrollment. Additional characterization of prevalent CAD included the CAD index $[12,13]$, an ordinal measure of location, and severity of CAD. Incident CVD events were defined as the composite endpoint of incident MI or need for therapeutic surgical (coronary artery bypass grafting or CABG) or endovascular interventions (percutaneous coronary intervention or PCI) during follow-up.

Genotyping

Consistent with previous studies [4], the 2 SNPs in the APOL1 G1 and the insertion/deletion polymorphism for the G2 risk allele were genotyped using Taqman genotyping assay (Life Technologies). Individuals' genotypes were categorized as noncarriers ( 0 or Ref/Ref), heterozygote carriers (1, G1/Ref, or G2/Ref), or homozygote carriers (2, G1/G1, G1/G2, or G2/G2). Details are described in the online supplementary material (www.karger.com/doi/10.1159/000453458). 
Wang et al.: Apolipoprotein L1 Genetic Variants Are Associated with CKD but Not with CVD in a Population Referred for Cardiac Catheterization

Table 1. Baseline characteristics of African-Americans in the CATHGEN study by APOL1 genotype, including renal disease and CVD

\begin{tabular}{|c|c|c|c|c|}
\hline Clinical characteristics & $\begin{array}{l}\text { Noncarriers } \\
(n=684)\end{array}$ & $\begin{array}{l}\text { Heterozygote carriers } \\
(n=726)\end{array}$ & $\begin{array}{l}\text { Homozygote carriers } \\
(n=231)\end{array}$ & $\begin{array}{l}p \\
\text { value* }^{*}\end{array}$ \\
\hline Mean age $\pm S D$, years & $57.8 \pm 11.6$ & $58.3 \pm 11.4$ & $55.7 \pm 11.6$ & 0.01 \\
\hline Male gender & $364(53.2)$ & 357 (49.2) & $130(56.3)$ & 0.11 \\
\hline Mean BMI \pm SD & $31.9 \pm 8.1$ & $32.3 \pm 8.5$ & $32.4 \pm 8.7$ & 0.65 \\
\hline Type 2 diabetes & $266(38.9)$ & $295(40.6)$ & $93(40.3)$ & 0.79 \\
\hline Hypertension & $516(75.4)$ & $570(78.5)$ & $188(81.4)$ & 0.13 \\
\hline Smoking & $303(44.3)$ & $293(40.4)$ & $104(45.0)$ & 0.24 \\
\hline Mean eGFR \pm SD & $77.7 \pm 28.9$ & $76.9 \pm 28.6$ & $67.9 \pm 35.9$ & $<0.001$ \\
\hline Prevalent CKD & $158(24.5)$ & 164 (23.9) & $75(33.5)$ & 0.01 \\
\hline On dialysis at baseline & $22(3.2)$ & $27(3.7)$ & $28(12.1)$ & $<0.001$ \\
\hline Incident CKD & $134(39.1)$ & $139(37.8)$ & $44(43.1)$ & 0.61 \\
\hline Prevalent CAD & $336(50.2)$ & $327(45.8)$ & $108(47.4)$ & 0.25 \\
\hline Median CAD index ${ }^{\mathrm{a}}(\mathrm{q} 1, \mathrm{q} 3)$ & $23(0,45)$ & $23(0,37)$ & $23(0,37)$ & 0.08 \\
\hline Number of diseased vessels & & & & 0.09 \\
\hline 0 & $333(49.8)$ & $387(54.2)$ & $120(52.6)$ & \\
\hline 1 & 119 (17.8) & $141(19.7)$ & $52(22.8)$ & \\
\hline 2 & $84(12.6)$ & $80(11.2)$ & $24(10.5)$ & \\
\hline 3 & $133(19.9)$ & $106(14.8)$ & $32(14.0)$ & \\
\hline Median LVEF (q1, q3) & $55(45,63)$ & $55(48,64)$ & $55(44,62)$ & 0.16 \\
\hline
\end{tabular}

Values are $n(\%)$ or means \pm SD, unless otherwise specified. $* p$ value by ANOVA or Kruskal-Wallis test.

${ }^{a} \mathrm{CAD}$ index: an ordinal measure of location and severity of CAD (0: no vessels with $>50 \%$ occlusion; 23 : $>1$ vessel with 50-74\% occlusion; 37: 2 diseased vessels).

\section{Statistical Analyses}

Genetic models were defined as additive $(0,1$, or 2 risk allele carriers), dominant ( 0 vs. 1 or 2 allelecarriers), or recessive ( 0 and 1 vs. 2 allele carriers). Clinical variables were compared among genotype groups using ANOVA or the Kruskal-Wallis test and the $\chi^{2}$ or the Fisher exact tests as appropriate. The clinical outcomes analyzed include: (1) prevalent CKD; (2) incident CKD; (3) prevalent CAD; (4) incident CVD events, and (5) overall survival or major adverse cardiovascular event-free (MACE-free) survival. Both basic (adjusted for age and sex) and full multivariable models [adjusted for age, sex, BMI, type 2 diabetes status, hypertension, smoking status, left ventricular ejection fraction (LVEF), and number of diseased vessels] were used. Associations between APOL1 variants and (1) prevalent CKD and (2) incident CKD were assessed under a recessive genetic model as established in previous studies $[2,5,10]$, while the association between APOL1 variants and CAD was assessed using recessive, dominant, and additive models. The Cox proportional hazards model was used to assess the associations between APOL1 variants and incident CVD events or mortality under an additive model. Overall and MACE-free survival was estimated using Kaplan-Meier methods. A subgroup analysis restricted to homozygote carriers was also conducted to examine potential clinical factors modifying the risk of incident CKD. All statistical analyses were conducted using the R software package version 3.2 (Vienna, Austria).

\section{Results}

The distribution of noncarriers, heterozygote carriers, and homozygote carriers of APOL1 risk alleles was $41.7 \%(n=722), 44.5 \%(n=771)$, and 13.8\% $(n=231)$, respectively.

Association between APOL1 Genotypes and Prevalent and Incident CKD

Table 1 demonstrates baseline clinical characteristics stratified by APOL1 genotype. Previously reported associations $[2,5,10]$ between the APOL1 risk variants and prevalent 


\section{CardioRenal Medicine}

Table 2. Clinical factors associated with incident CKD in African-Americans homozygous for APOL1 risk variants with normal eGFR at enrollment

\begin{tabular}{|c|c|c|c|c|}
\hline \multicolumn{5}{|l|}{ Cardiorenal Med 2017;7:96-103 } \\
\hline \multicolumn{2}{|l|}{ DOI: $10.1159 / 000453458$} & \multicolumn{3}{|c|}{$\begin{array}{l}\text { (C) } 2016 \text { S. Karger AG, Basel } \\
\text { www.karger.com/crm }\end{array}$} \\
\hline \multicolumn{5}{|c|}{$\begin{array}{l}\text { Wang et al.: Apolipoprotein L1 Genetic Variants Are Associated with CKD but Not with } \\
\text { CVD in a Population Referred for Cardiac Catheterization }\end{array}$} \\
\hline Variable & Estimate & OR & $95 \% \mathrm{CI}$ & $\begin{array}{l}p \\
\text { value }\end{array}$ \\
\hline Age (5-year increment) & 0.29 & 1.34 & $1.06-1.71$ & 0.02 \\
\hline Sex & 0.32 & 1.38 & $0.51-3.71$ & 0.52 \\
\hline BMI (5-point increment) & 0.27 & 1.31 & $0.99-1.76$ & 0.06 \\
\hline Type 2 diabetes & 1.00 & 2.71 & $1.09-7.03$ & 0.03 \\
\hline Hypertension & -0.44 & 0.64 & $0.17-2.26$ & 0.49 \\
\hline Smoking status & -0.52 & 0.60 & $0.23-1.47$ & 0.27 \\
\hline LVEF (5\% increment) & -0.15 & 0.86 & $0.74-0.98$ & 0.04 \\
\hline Number of diseased vessels & 0.11 & 1.12 & $0.72-1.73$ & 0.60 \\
\hline Baseline eGFR & -0.02 & 0.98 & $0.95-1.01$ & 0.18 \\
\hline
\end{tabular}

CKD were observed: the mean eGFR was significantly lower in homozygotes compared to heterozygotes and noncarriers (Table 1). APOL1 genotype was associated with prevalent CKD in the basic [odds ratio (OR): $1.85,95 \% \mathrm{CI}: 1.34-2.53, p=0.0002$ ] and full multivariable model (OR: 1.85, 95\% CI: 1.33-2.57, $p=0.0002$ ).

Information on incident CKD during follow-up was available for $813(49.5 \%)$ individuals of the study cohort. There was no significant association between APOL1 risk variants and incident CKD using either the basic or full multivariable logistic regression model (basic OR: 1.36, 95\% CI: 0.87-2.10, $p=0.17$; full OR: $1.28,95 \%$ CI: $0.80-2.03, p=0.30$ ).

\section{Subgroup Analysis}

Given the finding that 149 (66.5\%) homozygote carriers of APOL1 risk alleles had normal baseline eGFR, we explored analyses in this subgroup. Follow-up renal function was available in $102(68.5 \%)$ individuals of this subgroup, and 44 (43.1\%) subjects with normal baseline eGFR developed incident CKD during follow-up. Older age, presence of type 2 diabetes at baseline, and lower baseline LVEF were significant predictors of developing incident CKD (Table 2), with the presence of baseline type 2 diabetes being the strongest predictor, with an OR of 2.71 (95\% CI: 1.09-7.03, $p=0.03$ ). Further analysis to assess an interaction between APOL1 risk variants and type 2 diabetes at baseline in both heterozygote and homozygote carriers did not reveal a statistically significant interaction $(p=0.62)$.

Association between APOL1 Genotypes, CAD, Time to CVD Events, and All-Cause Mortality

No associations were detected between APOL1 variants and CAD (Table 3) or APOL1 variants and time to incident CVD events (heterozygote HR: $0.86,95 \% \mathrm{CI}: 0.63-1.19, p=0.38$; homozygote HR: $0.88,95 \%$ CI: $0.57-1.36, p=0.57$ ). Kaplan-Meier analysis revealed no significant difference in overall survival and MACE-free survival among noncarriers, heterozygote carriers, and homozygote carriers ( $p=0.98$ and $p=0.48$, respectively). Similarly, no significant association was detected between $A P O L 1$ risk variants and time to all-cause mortality (heterozygote HR: 1.1, 95\% CI: 0.87-1.30, $p=0.53$; homozygote HR: 0.89, 95\% CI: 0.67-1.19, $p=0.44)$.

\section{Discussion}

While the role of APOL1 risk variants in CKD has been established in previous studies [2, $4-6,10,14]$, our results expand the findings into a secondary cardiovascular prevention population for the first time using a large cohort of African-American individuals from a popu- 
Wang et al.: Apolipoprotein L1 Genetic Variants Are Associated with CKD but Not with CVD in a Population Referred for Cardiac Catheterization

Table 3. Association tests for APOL1 genotypes with prevalent CAD using additive, recessive, and dominant models

\begin{tabular}{|c|c|c|c|c|c|}
\hline Variable & Genetic model & Estimate & OR & $95 \% \mathrm{CI}$ & $p$ value \\
\hline \multirow[t]{2}{*}{ APOL1 heterozygote } & Additive, basic ${ }^{\mathrm{a}}$ & -0.18 & 0.84 & $0.67-1.04$ & 0.12 \\
\hline & Additive, multivariable ${ }^{\mathrm{b}}$ & -0.22 & 0.80 & $0.63-1.01$ & 0.06 \\
\hline \multirow[t]{4}{*}{ APOL1 homozygote } & Additive, basic ${ }^{\mathrm{a}}$ & -0.05 & 0.95 & $0.70-1.31$ & 0.77 \\
\hline & Additive, multivariable ${ }^{\mathrm{b}}$ & -0.98 & 0.91 & $0.65-1.26$ & 0.56 \\
\hline & Recessive, basic ${ }^{\mathrm{a}}$ & 0.04 & 1.05 & $0.78-1.40$ & 0.77 \\
\hline & Recessive, multivariable ${ }^{b}$ & 0.016 & 1.02 & $0.75-1.38$ & 0.92 \\
\hline \multirow{2}{*}{$\begin{array}{l}\text { APOL1 homozygote or } \\
\text { heterozygote carriers }\end{array}$} & Dominant, basic ${ }^{\mathrm{a}}$ & -0.15 & 0.86 & $0.70-1.06$ & 0.17 \\
\hline & Dominant, multivariable ${ }^{\mathrm{b}}$ & -0.19 & 0.82 & $0.66-1.03$ & 0.08 \\
\hline
\end{tabular}

${ }^{a}$ Adjusted for age and sex. ${ }^{b}$ Adjusted for age, sex, BMI, type 2 diabetes status, hypertension status, smoking status, and baseline eGFR.

lation with high cardiometabolic risk. Besides renal disease, we assessed the association of APOL1 risk variants with CVD and did not demonstrate a significant relationship. Interestingly, we found that $66.5 \%$ of African-Americans presumed at risk of CKD based on their APOL1 homozygous background had normal eGFR at the time of cardiac catheterization, prompting analyses demonstrating that age, type 2 diabetes, and baseline LVEF are factors influencing the risk of subsequent CKD in this at-risk population.

Our results suggest that incident CKD is a heterogeneous and multifactorial disease, even in individuals at high risk due to an APOL1 homozygous background. Previous studies have alluded to the presence of other genetic and environmental factors that could contribute to the risk for CKD by interacting with APOL1 [7, 15-17]. In a recent analysis of the AfricanAmerican Study of Kidney Disease and Hypertension (AASK) [16], many sociodemographic (age $>55$ years, sex, education, income, etc.) and common clinical risk factors (systolic blood pressure, BMI, smoking, etc.) were examined, but none were shown to alter APOL1-related CKD progression.

In our study, older age, presence of type 2 diabetes, and lower LVEF at baseline are 3 factors that predicted incident CKD development in an APOL1 homozygous background. Although the association between older age and renal function decline has been reported previously [18, 19], few studies have reported the effect of age on incident CKD in an APOL1 background. In the study by Garg et al. [18], the authors found that each 5-year increase in age was associated with an increase of $0.01-0.1 \mathrm{mg} / \mathrm{dL}$ in serum creatinine. In another study that examined mortality risk stratification in CKD [19], the prevalence of CKD increased from 3 to $49 \%$ as age increased from 18-44 to 85-100 years. In the recent study by Chen et al. [16], age was not a significant modifier of the association between APOL1 risk alleles and CKD progression. Our study differed from the AASK study in that we examined age in 5-year increments, instead of a binary variable with the cutoff at 55 years, which may have contributed to the difference in results.

A lower LVEF at baseline was another factor that predicted incident CKD development in an APOL1 homozygous background. Most previous studies on low EF and CKD have focused on worsening renal function in heart failure [20-22]. Lower EF has been shown to predict worsening renal function ( $p=0.002$ ) in some previous studies [20], but not others [21]. The mechanisms responsible for renal decline in the setting of low EF may include decreased renal perfusion secondary to low cardiac output and neurohormonal activation [20]. 
In this study, the presence of type 2 diabetes was the strongest and only modifiable factor that increased the risk of CKD in individuals with an APOL1 homozygous background. Few previous studies on APOL1 included patients with diabetes $[15,16]$. In studies that did, a stronger association between APOL1 risk variants and renal decline has been shown in this subgroup [4]. For example, the Chronic Renal Insufficiency Cohort (CRIC) study found that over a mean follow-up of 4.4 years, African-American individuals with homozygous APOL1 without diabetes experienced renal events at a rate of 7.5 per 100 personyears, compared with an event rate of 13.7 per 100 person-years in individuals with diabetes [4].

Potential mechanisms for the increased risk conferred by diabetes in an APOL1 high-risk background are poorly understood, partially because the underlying mechanisms by which APOL1 risk alleles lead to renal disease are unclear. A recent in vitro study investigated the effects of overexpressing nonrisk and risk variants of APOL1 in human podocytes through a lentivirus expression system and discovered that APOL1 risk variants induced podocyte necrosis through compromising lysosomal membrane permeability [23]. The same study also identified hypoxia as an environmental modifier that enhanced the effects of APOL1 risk variants. Hypoxia secondary to the mismatch of oxygen demand and delivery [24] is a common finding in type 2 diabetes, and may be the underlying mechanism explaining type 2 diabetes status as the strongest predictor of incident CKD in this subgroup. Other potential mechanisms could include the role of diabetes in renal damage itself, such as glycation end products causing mesangial expansion [25].

The lack of association between APOL1 and CVD discovered in our study has been observed in other large trial-based studies, including the AASK and the Systolic Blood Pressure Intervention Trial (SPRINT) [4, 5]. However, these results are in contrast with the JHS and WHI study [6]. Possible explanations for these discrepancies include differences in patient selection, definition of clinical phenotypes, and censoring methods.

Our study has limitations. The follow-up for incident CKD was not available in some patients, which could introduce biases. Additionally, we were unable to determine the exact cause of incident CKD in this study. Other potential factors associated with risk of incident CKD, such as blood pressure control and medications, were not included in our analysis. However, these factors were not shown to affect APOL1-related CKD progression in the recent study of the AASK population [16]. Detailed cardiovascular follow-up also allowed us to use true time-to-event information, as opposed to using nested cases and controls, to assess the associations between APOL1 risk variants and incident CVD events and survival.

\section{Conclusions}

We confirmed that APOL1 G1 and G2 variants are associated with prevalent renal disease in African-Americans, and expanded these findings into a secondary prevention population. We found no association between APOL1 risk variants and CAD or CVD events. Building a clinical model to identify factors increasing the risk of incident CKD in APOL1 homozygous individuals, we demonstrated that the causes for incident CKD are multifactorial. Type 2 diabetes was the strongest and only modifiable factor that predicted incident CKD in this subgroup. These results highlight the need for further studies on the potential modifying effects of diabetes control on CKD development and studies to understand the potential interactive mechanisms between APOL1 and diabetes on CKD. 


\section{CardioRenal Medicine}

\begin{tabular}{l|l}
\hline Cardiorenal Med 2017;7:96-103 \\
\hline DOI: 10.1159/000453458 & $\begin{array}{l}\text { (C) 2016 S. Karger AG, Basel } \\
\text { www.karger.com/crm }\end{array}$ \\
\hline Wang et al.: Apolipoprotein L1 Genetic Variants Are Associated with CKD but Not with
\end{tabular}

CVD in a Population Referred for Cardiac Catheterization

\section{Statement of Ethics}

All subjects in the CATHGEN study have given their informed written consent. The study protocol was approved by the Institutional Review Board (IRB) of Duke University. Funding for this study was supported by the National Institutes of Health Grant HL095987 (S.H.S.); National Institute of Diabetes, Digestive and Kidney Diseases of the National Institutes of Health under Award No. P30DK096493 (S.H.S.), and T32HL007101 (H.W.).

\section{Disclosure Statement}

The authors have no conflicts of interest to disclose.

\section{References}

1 Saab KR, Kendrick J, Yracheta JM, Lanaspa MA, Pollard M, Johnson RJ: New insights on the risk for cardiovascular disease in African Americans: the role of added sugars. J Am Soc Nephrol 2015;26:247-257.

2 Genovese G, Friedman DJ, Ross MD, Lecordier L, Uzureau P, Freedman BI, et al: Association of trypanolytic ApoL1 variants with kidney disease in African-Americans. Science 2010;329:841-845.

3 Foster MC, Coresh J, Fornage M, Astor BC, Grams M, Franceschini N, et al: APOL1 variants associate with increased risk of CKD among African Americans. J Am Soc Nephrol 2013;24:1484-1491.

4 Parsa A, Kao WHL, Xie D, Astor BC, Li M, Hsu C, et al: APOL1 risk variants, race, and progression of chronic kidney disease. N Engl J Med 2013;369:2183-2196.

5 Langefeld CD, Divers J, Pajewski NM, Hawfield AT, Reboussin DM, Bild DE, et al: Apolipoprotein L1 gene variants associate with prevalent kidney but not prevalent cardiovascular disease in the Systolic Blood Pressure Intervention Trial. Kidney Int 2014;87:169-175.

6 Ito K, Bick AG, Flannick J, Friedman DJ, Genovese G, Parfenov MG, et al: Increased burden of cardiovascular disease in carriers of APOL1 genetic variants. Circ Res 2014;114:845-850.

7 Friedman DJ, Pollak MR: Genetics of kidney failure and the evolving story of APOL1. J Clnical Investig 2011; 121:3367-3374.

8 Freedman BI, Langefeld CD, Lu L, Palmer ND, Carrie Smith S, Bagwell BM, et al: APOL1 associations with nephropathy, atherosclerosis, and all-cause mortality in African Americans with type 2 diabetes. Kidney Int 2014;87:176-181.

9 Kraus WE, Granger CB, Sketch MH, Donahue MP, Ginsburg GS, Hauser ER, et al: A Guide for a Cardiovascular Genomics Biorepository: the CATHGEN Experience. J Cardiovasc Transl Res DOI: 10.1007/s12265-0159648-y.

10 Tzur S, Rosset S, Shemer R, Yudkovsky G, Selig S, Tarekegn A, et al: Missense mutations in the APOL1 gene are highly associated with end stage kidney disease risk previously attributed to the MYH9 gene. Hum Genet 2010; 128:345-350.

11 Levey AS, Stevens LA, Schmid CH, Zhang YL, Iii AFC, Feldman HI, et al: A new equation to estimate glomerular filtration rate. Ann Intern Med 2009;150:604-612.

12 Mark DB, Nelson CL, Califf RM, Harrell FE, Lee KL, Jones RH, et al: Continuing evolution of therapy for coronary artery disease. Initial results from the era of coronary angioplasty. Circulation 1994;89:2015-2025.

13 Shah SH, Freedman NJ, Zhang L, Crosslin DR, Stone DH, Haynes C, et al: Neuropeptide Y gene polymorphisms confer risk of early-onset atherosclerosis. PLoS Genet 2009;5:e1000318.

14 Tzur S, Rosset S, Skorecki K, Wasser WG: APOL1 allelic variants are associated with lower age of dialysis initiation and thereby increased dialysis vintage in African and Hispanic Americans with non-diabetic end-stage kidney disease. Nephrol Dial Transplant 2012;27:1498-1505.

15 Divers J, Palmer ND, Lu L, Langefeld CD, Rocco MV, Hicks PJ, et al: Gene-gene interactions in APOL1-associated nephropathy. Nephrol Dial Transplant 2014;29:587-594.

16 Chen TK, Choi MJ, Kao WHL, Astor BC, Scialla JJ, Appel LJ, et al: Examination of potential modifiers of the association of APOL1 alleles with CKD progression. Clin J Am Soc Nephrol 2015;10:1-8.

17 Tin A, Grams ME, Maruthur NM, Astor BC, Couper D, Mosley TH, et al: Hemostatic factors, APOL1 risk variants, and the risk of ESRD in the Atherosclerosis Risk in Communities Study. Clin J Am Soc Nephrol 2015;10:1-7.

18 Garg A, Papaioannou A, Ferko N: Estimating the prevalence of renal insufficiency in seniors requiring longterm care. Kidney Int 2004;65:649-653.

19 O'Hare AM: Mortality risk stratification in chronic kidney disease: one size for all ages? J Am Soc Nephrol 2006; 17:846-853.

20 Metra M, Nodari S, Parrinello G, Bordonali T, Bugatti S, Danesi R, et al: Worsening renal function in patients hospitalised for acute heart failure: clinical implications and prognostic significance. Eur J Heart Fail 2008;10: 188-195. 
21 Forman DE, Butler J, Wang Y, Abraham WT, O'Connor CM, Gottlieb SS, et al: Incidence, predictors at admission, and impact of worsening renal function among patients hospitalized with heart failure. J Am Coll Cardiol 2004; 43:61-67.

22 Krumholz HM, Chen Y-T, Vaccarino V, Wang Y, Radford MJ, Bradford WD, et al: Correlates and impact on outcomes of worsening renal function in patients $\geq 65$ years of age with heart failure. Am J Cardiol 2000;85: 1110-1113.

23 Lan X, Jhaveri A, Cheng K, Wen H, Saleem MA, Mathieson PW, et al: APOL1 risk variants enhance podocyte necrosis through compromising lysosomal membrane permeability. Am J Physiol Renal Physiol 2014; 307:F326-F336.

24 Hansell P, Welch WJ, Blantz RC, Palm F: Determinants of kidney oxygen consumption and their relationship to tissue oxygen tension in diabetes and hypertension. Clin Exp Pharmacol Physiol 2013;40:123-137.

25 Abe H, Tominaga T, Abe N, Kishi S, Murakami T, Araoka T: Scleraxis modulates bone morphogenetic protein 4 (BMP4)-Smad1 protein-smooth muscle $\alpha$-actin (SMA) signal transduction in diabetic nephropathy. J Biol Chem 2012;287:20430-20442. 